\title{
Endocrinological Analysis of 122 Japanese Childhood Cancer Survivors in a Single Hospital
}

\author{
YOKO MIYOSHI, HIDEAKI OHTA, YOSHIKO HASHII, SADAO TOKIMASA, NORIYUKI NAMBA, \\ SOTARO MUSHIAKE, JUNICHI HARA* AND KEIICHI OZONO
}

Department of Pediatrics, Osaka University Graduate School of Medicine, Osaka, Japan

*Department of Pediatric Hematology/Oncology, Osaka City General Hospital, Osaka, Japan

\begin{abstract}
With recent improvements in the diagnosis and treatment of cancer, the number of childhood cancer survivors (CCSs) has been increasing in Japan. The importance of quality of life during the lifetime of CCSs has now been recognized, and the late effects of cancer treatments are essential and important issues. In this study we analyzed the endocrinological abnormalities of CCSs by retrospectively evaluating 122 outpatients (62 males and 60 females) who had been referred from pediatric oncologists to our follow-up clinic among 151 CCSs attending our hospital more than two years after their cancer treatment. Follow-up duration varied from 2 to 30 (median 8.0) years. Their average age was 17.3 (range 4-36, median 17.0) years, and 38 patients (31.1\%) reached adulthood. Endocrinological abnormalities were found in $82(67 \%)$ of 122 survivors. Gonadal dysfunction was observed in 60 patients $(49 \%)$. Thirty-nine patients $(32 \%)$ were short or grew at a slower rate. Twenty-six patients $(21 \%)$ showed thyroid dysfunction. Other abnormalities were as follows: obesity in 20 patients (16\%), leanness in $10(8 \%)$, central diabetes insipidus in $11(9 \%)$ and adrenocortical dysfunction in $9(7 \%)$. Low bone mineral density was observed in $41(42 \%)$ of 98 patients evaluated. These endocrinological abnormalities were caused by the combined effects of cancer itself and various treatments (chemotherapy, radiation therapy, surgery, and hematopoietic stem cell transplantation). Lifetime medical surveillance and continuous follow-up are necessary for CCSs, because treatment-related complications may occur during childhood and many years after the therapy as well. Endocrinologists should participate in long-term follow-up of these survivors in collaboration with pediatric oncologists.
\end{abstract}

Key words: Childhood cancer survivors, Late effects, Endocrinological abnormality, Growth retardation, Quality of life (Endocrine Journal 55: 1055-1063, 2008)

WITH recent improvements in the diagnosis and treatment of children with cancer, the number of childhood cancer survivors (CCSs) is surmised to be rapidly growing in Japan as well as other countries, including the U.S.A. and Europe [1-3]. Approximately $80 \%$ of children with cancer now survive longer than five years $[2,3]$, however, this improved survival has been accompanied by the occurrence of late treatment-related complications, late effects, such as second neoplasm, organ dysfunctions and psychosocial problems, which

Received: March 11, 2008

Accepted: August 6, 2008

Correspondence to: Keiichi OZONO, M.D., Ph.D., Department of Pediatrics, Osaka University Graduate School of Medicine, Suita City, Yamadaoka 2-2, Osaka, 565-0871, Japan have become essential and important issues [4-7]. Among late effects, endocrinological abnormalities are the most common problems, often requiring early interventions $[8,9]$. Several reports from the U.S.A. and Europe have described the late effects or adverse health outcomes in a large number of CCSs, and guidelines to follow-up CCSs from abroad are now available [3, 10-14], however, only a few studies on CCSs, especially focused on endocrinological abnormalities, have been published in Japan $[15,16,17]$. The aim of the present retrospective study was to assess the endocrine abnormalities of CCSs referred from pediatric oncologists to our follow-up clinic in our hospital situated in the western Japan. 
Table 1. Patient profile and endocrinological late effects of $122 \mathrm{CCSs}$

\begin{tabular}{|c|c|c|c|c|c|}
\hline & & $\begin{array}{c}\text { Total } \\
(\mathrm{n}=122)\end{array}$ & $\begin{array}{l}\text { Hematologial } \\
\quad(\mathrm{n}=67)\end{array}$ & $\begin{array}{l}\text { Brain tumor } \\
\quad(\mathrm{n}=26)\end{array}$ & $\begin{array}{l}\text { Solid tumor } \\
\quad(\mathrm{n}=29)\end{array}$ \\
\hline \multirow[t]{6}{*}{ Therapy } & Chemotherapy & $116(95 \%)$ & 67 & 20 & 29 \\
\hline & Surgery & $57(47 \%)$ & 5 & 23 & 29 \\
\hline & Radiation therapy & $72(59 \%)$ & 42 & 20 & 10 \\
\hline & HSCT & $65(53 \%)$ & 36 & 10 & 19 \\
\hline & Conditioning: TBI & 25 & 25 & 0 & 0 \\
\hline & Conditioning: non-TBI & 40 & 11 & 10 & 19 \\
\hline \multirow{10}{*}{$\begin{array}{l}\text { Endocrine } \\
\text { disorder }\end{array}$} & Total & $82(67 \%)$ & $39(58 \%)$ & $25(96 \%)^{*}$ & $18(62 \%)$ \\
\hline & Growth disturbance & $39(32 \%)$ & 14 & $17 *$ & 8 \\
\hline & Obesity & $20(16 \%)$ & 11 & 6 & 3 \\
\hline & Leanness & $10(8 \%)$ & 5 & 1 & 4 \\
\hline & Hypogonadism & $60(49 \%)$ & 23 & $20^{*}$ & 17 \\
\hline & Primary & $51(\mathrm{M} \mathrm{26,} \mathrm{F} \mathrm{25)}$ & 23 (M 11, F 12) & 11 (M 5, F 6) & 17 (M 10, F 7) \\
\hline & Central & 9 (M 4, F 5) & 0 & $9(\mathrm{M} 4, \mathrm{~F} 5)$ & 0 \\
\hline & Thyroid dysfunction & $26(21 \%)$ & 8 & $16^{*}$ & 2 \\
\hline & Adrenocortical dysfunction & $9(7 \%)$ & 0 & $9^{*}$ & 0 \\
\hline & Central diabetes insipidus & $11(9 \%)$ & 0 & $10^{*}$ & 1 \\
\hline
\end{tabular}

* Significant high incidence: $\mathrm{p}<0.01$ by chi square test

\section{Patients and methods}

\section{Patients}

The records of 151 CCSs who had survived for more than two years after cancer treatment and been followed regularly in Osaka University Hospital were reviewed, retrospectively. This study analyzed 122 patients (62 males and 60 females), who had been referred from oncologists to our follow-up group among 151 CCSs in our pediatric department from January 1997 to December 2007. The unreferred 29 patients were excluded in the study: hematological disease $(n=6)$, brain tumor $(n=5)$ and solid tumor $(n=18)$. Follow-up duration varied from 2 to 30 (mean 8.8, median 8.0) years. Average age was 17.3 (range 4-36, median 17.0) years, and 38 patients $(31 \%)$ reached adulthood. Mean age at diagnosis of the underlying disease was 6.4 (range $0-15$ ) years. The underlying disease and treatment regimens [chemotherapy, radiation therapy, surgery and hematopoietic stem cell transplantation (HSCT)] were assessed from the medical records. Table 1 lists the patient profiles. Diagnosis was hematological disease (67), brain tumor (26) and solid tumor (29). Hematological malignant diseases were classified as follows: acute lymphoblastic leukemia (34), acute myelocytic leukemia (12), nonHodgkin lymphoma (8), chronic myelocytic leukemia
(3), juvenile myelomonocytic leukemia (2), Hodgkin lymphoma (2) and myelodysplastic syndrome (1). Nonmalignant hematological diseases included aplastic anemia (3), Wiskott-Aldrich syndrome (1) and leukocyte adhesion deficiency (1). Brain tumors were classified as germinoma (11), medulloblastoma (9) and craniopharyngioma (6). Solid tumors were classified as neuroblastoma (7), rhabdomyosarcoma (8), hepatoblastoma (4), primitive neuroectodermal tumor (2), Wilms' tumor (2), germinoma (1), upper pharyngeal tumor (1), teratoma (1), eosinophilic granuloma (1), ovarian undifferentiated germinoma (1) and granulosa cell tumor of the ovary (1). Number in each parenthesis indicates the number of patients.

\section{Treatment}

Treatment protocols for cancer have changed over the years. The treatment regimens of the 122 survivors included 116 chemotherapy (95\%), 72 radiotherapy $(59 \%)$ and 57 surgery (47\%). Sixty-five patients (53\%) underwent HSCT, including 33 allogeneic and 32 autologous sources. Five patients underwent two transplants, and one patient, three transplants. Hematopoietic stem cells were derived from bone marrow $(n=45)$, peripheral blood $(n=23)$ and umbilical cord blood $(n=3)$. Twenty-five patients with hematological diseases received radiation as a conditioning 
for HSCT: total body irradiation (TBI) at a dose of 8 12 Gy in 4 to 8 fractions in 22 patients, TBI without cranial irradiation at a dose of 6-10 Gy in 2 to 4 fractions in 2 patients and total lymphoid irradiation at a dose 6 Gy in 3 fractions in 1 patient. The conditioning regimen for HSCT in the other 40 patients was chemotherapy only, including $8-16 \mathrm{mg} / \mathrm{kg}$ of busulfan and/or $100-200 \mathrm{mg} / \mathrm{kg}$ of cyclophosphamide for hematological disease, or $800 \mathrm{mg} / \mathrm{m}^{2}$ of thio-TEPA and $280 \mathrm{mg}$ / $\mathrm{m}^{2}$ of melphalan for brain tumor and solid tumor. The dose of radiation varied with the case: 3-24 Gy to the whole brain for hematological diseases, 10-54 Gy to the local cerebral region plus $18-30$ Gy to the whole brain with 18-32 Gy to the whole spine for brain tumors and 20-45 Gy to the local lesion for solid tumors.

\section{Evaluation methods}

The current physical and psychological status, use of medication and school health check were inquired from patients and their parents. Height and weight were measured at every visit. Current height was expressed as the standard deviation score (SDS) for age and gender. Short stature was defined as a stature $<-2$ $\mathrm{SD}$ for chronological age or slow growth as growth velocity 1.5 SD below the mean for more than two years. Patients under GH therapy were all included in this category. GHD (growth hormone deficiency) was defined as a low IGF-1 level and low peak GH level on the GH provocation tests. Subjects with peak GH levels $<10 \mathrm{ng} / \mathrm{ml}$ on insulin, arginine, clonidine and dopamine were considered to have abnormal GH secretion. Obesity was defined as $>20 \%$ obese for ideal weight, and leanness as $<20 \%$ lean for ideal weight. Pubertal status was assessed by the method of Tanner. Serum levels of basal FSH, LH and testosterone (boys)/estradiol (girls) were measured. For boys, testicular volume was determined using an orchidometer. Failure of spontaneous puberty was defined as the absence of breast development in girls at 14 years of age or more and of testicular enlargement in boys at 15 years of age or more. Tubular damage of male patients was defined by little $(<10 \mathrm{ml})$ [16] or no increase in testicular volume combined with a high basal FSH level $(>20 \mathrm{mIU} / \mathrm{ml})$ at pubertal age. For girls, the age at initiation and regularity of menstruation were inquired. FSH and LH values were considered high or low in primary and central hypogonadism. The cut-off point for early pubertal signs was 9 years of age for boys and 7.5 years for girls. Thyroid status was evaluated by palpation of the thyroid gland, and also measurement of serum TSH and FT4 levels. Primary hypothyroidism was defined as $\mathrm{TSH} \geq 10 \mu \mathrm{U} / \mathrm{ml}$ and central hypothyroidism as FT4 $<0.8 \mathrm{ng} / \mathrm{dl}$ and inappropriately elevated TSH. In hypothyroidism, antithyroid peroxidase (TPO) antibody and/or anti-thyroglobulin (Tg) antibody were measured. Adrenocortical function was assessed by serum ACTH and cortisol levels and serum DHEAS. These endocrine tests were measured at outpatient clinic in the morning to avoid diurnal variation of hormones. Central diabetes insipidus was defined as polyuria and necessity for DDAVP therapy.

\section{Bone mineral density}

BMD (bone mineral density) $\left(\mathrm{g} / \mathrm{cm}^{2}\right)$ of the lumbar spine (L2-L4) was measured by dual energy X-ray absorptiometry (DXA) in 98 out of 122 patients: DPX-L (LUNAR) until March 2006 and Discover QDR 1000 (HOLOGIC) after April 2006. The conversion formula was as follows: [QDR 1000] $=0.827 \times[\mathrm{DPX}-$ L] +0.042 . In adult cases, osteopenia was defined as between 70 and $80 \%$ of the Japanese young adult mean (YAM) and osteoporosis as less than $70 \%$ of YAM [18]. The BMD in childhood was expressed as age-normalized Z-scores (SD from the mean for ageand sex-matched controls) according to the report by Tanaka [19]. In children, osteopenia was defined as a Z-score between 1.7 and 2.6 SD below the mean, and osteoporosis as a Z-score of more than 2.6 SD below the mean.

\section{Statistical Analysis}

The chi-square test was used to assess the difference between each group in Table 1 and Table 3. The twotail Wilcoxon t-test was used to compare the differences of mean height-SDS in Table 2. These statistical analyses were carried out using Excel Ystat 2006.

\section{Results}

Endocrinological abnormalities were found in 82 (67\%) of 122 CCSs in our follow-up clinic: 39 (58\%) of 67 patients with hematological diseases, 25 (96\%) 
of 26 patients with brain tumors and $18(62 \%)$ of 29 patients with solid tumors. Frequency of endocrinological disorders was significantly higher in patients with brain tumors compared to other types of tumors (Table 1, $\mathrm{p}<0.01$ ). Hormone replacement therapies were prescribed as follows: sex hormone $(n=29$ : male 7 , female 22), levothyroxine $(\mathrm{n}=22), \mathrm{GH}(\mathrm{n}=13)$, DDAVP $(n=10)$, and corticosteroid $(n=9)$. These endocrinological problems have emerged at different sites and times on a case-by-case basis even when common protocols were used for the same diseases.

\section{Physical growth}

Thirty-nine patients (32\%) had a height $<-2$ SD for age or slow growth at the last assessment. Sixteen patients were diagnosed with GHD, and 13 patients had been treated with recombinant human GH (Table 2). The average age at GH initiation was 11.6 years old (range, 5.4 to 14.6), 5.3 years after the end of cancer treatment (range, 1.5 to 11.3 years). Mean height-SDS at initiation of GH therapy was -3.4 (range, -6.2 to -2.2 ), and current height-SDS was -1.8 SD (range, -5.0 to the mean). Height gain was significant according to these improved SD scores as a group $(p<0.01)$. However, a few cases with delayed or short duration of GH therapy showed poor improvement in height. We started sex hormone replacement therapies at relatively late ages to avoid closure of growth plate and to ensure longer duration of GH therapy. GH therapy was stopped in two patients: one patient (case 3) with germinoma due to poor compliance and an abnormal oral glucose tolerance test, and an extremely obese patient (case 8) with craniopharyngioma due to the occurrence of type 2 diabetes mellitus. In the last evaluation, 20 $(16 \%)$ of 122 patients were obese, and 10 patients $(8 \%)$ were lean for their ideal weight.

\section{Thyroid status}

Twenty-six patients (21\%) were diagnosed with thyroid dysfunctions, and $22(18 \%)$ were treated with levothyroxine replacement therapy among 122 CCSs. Sixteen patients had primary hypothyroidism and 9 patients had central hypothyroidism. One patient (meduloblastoma), who had received radiation therapy to the brain and spine, alternately showed both types of hypothyroidism. Anti-Tg antibody and/or anti-TPO antibody were positive in 6 patients; 4 had allogeneic transplantations. Nine of 10 patients with primary hypothyroidism who had no anti-thyroid autoantibody had been irradiated to the neck for their underlying disease or as a conditioning regimen for HSCT. All 9 patients with central hypothyroidism had been treated for suprasellar brain tumors, 3 germinoma and 6 craniopharyngioma. No case of hyperthyroidism or thyroid cancer was seen in our study.

\section{Gonadal function and puberty}

\section{Males}

Thirty (48\%) pubertal boys out of 62 male CCSs had abnormal gonadal functions: 4 with central hypogonadism and 26 with primary hypogonadism. All 4 patients with central hypogonadism manifested panhypopituitarism after the therapy of suprasellar brain tumors. All 26 primary hypogonadic males, 11 patients with hematological diseases, 5 with brain tumors and 10 with solid tumors, had been treated with chemotherapy including alkylating agent, cyclophosphamide. Eight of 11 patients with hematological diseases had also been treated with TBI for HSCT. Most male patients with primary hypogonadism had begun spontaneous puberty. Sex hormone replacement therapy was administered to only 7 patients, 4 with central hypogonadism and 3 with primary hypogonadism. It was difficult to identify gonadal dysfunctions in prepubertal patients. Sperm evaluations were not routinely performed. Three males with $\beta$-HCG-producing germinoma presented with precocious puberty and advanced bone age at diagnosis. Their accelerated puberty was stopped when cancer treatment was initiated, and they showed primary hypogonadism after chemotherapy including cyclophosphamide.

\section{Females}

Thirty (50\%) out of 60 female CCSs were found to have abnormal gonadal functions: 5 with central hypogonadism and 25 with primary hypogonadism. All 5 patients with central hypogonadism had suprasellar brain tumors. Among 25 hypogonadic females, 12 patients with hematological diseases, 6 with brain tumors and 7 with solid tumors, had been treated with chemotherapy including alkylating agent, cyclophosphamide. Nine of 12 female patients with hematological diseases had also been treated with TBI for HSCT. Sex hormone replacement therapy was administered to $22(19$ primary, 3 central) hypogonadic patients. These re- 
Table 2. Patient profiles of the 13 CCSs treated with GH therapy

\begin{tabular}{rlccccccccccc}
\hline Case & \multicolumn{1}{c}{ Disease } & Age & Sex & $\begin{array}{c}\text { Age (yr) at } \\
\text { diagnosis }\end{array}$ & CT & Surgery & RT & HSCT & $\begin{array}{c}\text { Age (yr) } \\
\text { at GH start }\end{array}$ & $\begin{array}{c}\text { Height-SDS } \\
\text { at GH start }\end{array}$ & $\begin{array}{c}\text { Height-SDS } \\
\text { at present }\end{array}$ & $\begin{array}{c}\text { Age (yr) } \\
\text { at HRT start }\end{array}$ \\
\hline 1 & ALL & 13 & F & 0 & + & - & + & + & 8.5 & -2.7 & -2.9 & 12 \\
2 & AML & 18 & F & 1 & + & - & - & + & 14.6 & -2.9 & $-2.4^{*}$ & - \\
\hline 3 & Germinoma & 15 & F & 11 & + & - & + & - & 14.1 & -3.8 & $-2.5^{*}$ & not yet \\
4 & Germinoma & 16 & F & 9 & + & + & + & + & 13.9 & -3.2 & $-1.5^{*}$ & 15 \\
5 & Craniopharyngioma & 11 & F & 5 & - & + & - & - & 9.7 & -2.2 & -1.1 & not yet \\
6 & Craniopharyngioma & 13 & M & 3 & - & + & - & - & 5.4 & -2.4 & -0.6 & 13 \\
7 & Craniopharyngioma & 17 & M & 11 & - & + & - & - & 14.1 & -2.5 & -0.3 & 15 \\
8 & Craniopharyngioma & 21 & F & 11 & - & + & - & - & 13.1 & -3.7 & $-1.3^{*}$ & 16 \\
9 & Craniopharyngioma & 32 & F & 1 & - & + & - & - & 6.1 & -5.8 & Mean* & 15 \\
10 & Medulloblastoma & 12 & F & 7 & + & - & + & - & 11.3 & -2.5 & -2.1 & - \\
11 & Medulloblastoma & 15 & F & 4 & + & + & + & + & 13.8 & -6.2 & -5.0 & not yet \\
\hline 12 & Hepatoblastoma & 13 & F & 0 & + & + & - & + & 10.8 & -2.6 & -2.6 & not yet \\
13 & Neuroblastoma & 17 & F & 2 & + & + & - & + & 13.7 & -3.2 & -1.0 & 16 \\
\hline
\end{tabular}

CT, chemotherapy; RT, radiotherapy; HSCT, hematopoietic stem cell transplantation; SDS, standard deviation score; HRT, hormone replacement therapy for hypogonadism

* GH therapy stopped

placement therapies were initiated relatively late at pubertal age for the purpose of height gain as mentioned above. Although one girl showed transient normalization of $\mathrm{FSH} / \mathrm{LH}$, none of the girls with ovarian failure recovered to normal function. One girl showed central precocious puberty, and two girls showed borderline age of pubertal initiation. These three girls had been treated with 15-18 Gy cranial irradiation therapy at the age of three.

\section{Bone mineral density}

BMD $\left(\mathrm{g} / \mathrm{cm}^{2}\right)$ of the lumbar spine (L2-L4) in 98 (44 males and 54 females) of 122 patients was measured. Low BMD was observed in 41 patients $(42 \%)$ : thirty patients (16 males and 14 females) showed osteopenia (31\%), and 11 patients ( 2 males and 9 females) showed osteoporosis (11\%). Nine $(36 \%)$ out of 25 adult patients showed decreased BMD. Twenty-one $(58 \%)$ of 36 short stature patients and $7(58 \%)$ of 12 GHD showed low bone density. Eight $(35 \%)$ of 23 hypogonadic males and 17 (57\%) of 30 hypogonadic females also showed low BMD. Among 11 extremely low BMD group, namely osteoporosis patients, 9 $(82 \%)$ patients had short stature, $5(45 \%)$ had GHD, and $10(91 \%)$ had hypogonadism. Although the number of osteoporosis patients is small, growth disturbance, GHD and hypogonadism were considered to be risk factors for low BMD (Table 3). One male with chronic graft-versus-host disease (GVHD) experienced compressed fracture of the lumbar spine.

\section{Other endocrine abnormalities}

Nine $(7 \%)$ patients with suprasellar brain tumor showed panhypopituitarism including adrenocortical dysfunction. All were treated with corticosteroid replacement therapy. Central diabetes insipidus was demonstrated in 11 (9\%) patients: craniopharyngioma $(n=6)$, germinoma $(n=4)$ and Langerhans cell histiocytosis $(n=1)$. DDAVP replacement therapy was stopped in one patient with craniopharyngioma due to improved symptoms two years after the operation.

\section{Discussion}

As the survival rates for childhood cancers have improved, the importance of quality of life over a long lifetime and the late effects of CCSs have become recognized [20]. Cure and care of long-term survivors of childhood cancer has been recently reported from abroad [21] and a high rate of illness affecting chronic health has been documented $[5,6]$. In the present study, we investigated late effects, especially endocrinological complications of CCSs in a single Japanese hospital. The endocrine organs are sensitive to both chemotherapy and radiation. Endocrine disturbance have been documented in $20 \%$ to $50 \%$ of CCSs [22]. 
Table 3. Lumbar bone mineral density of $98 \mathrm{CCSs}$

\begin{tabular}{lccc}
\hline & No. of patients evaluated & Low BMD & Osteoporosis \\
\hline \multicolumn{1}{c}{ Total } & 98 (M 44, F 54) & 41 (M 18, F 23) & 11 (M 2, F 9) \\
\hline Hematological & 54 & 23 & 7 \\
Brain tumor & 24 & 11 & 3 \\
Solid tumor & 20 & 7 & 1 \\
\hline Adulthood & 25 & 9 & 1 \\
\hline Radiation & 66 & 29 & 9 \\
Post HSCT & 60 & $32^{*}$ & 9 \\
\hline Growth disturbance & 36 & $21^{* *}$ & $9 *$ \\
GHD & 12 & 7 & $5^{*}$ \\
Hypogonadism & 53 (M 23, F 30) & 25 (M 8, F 17) & 10 (M 1, F 9)** \\
\hline
\end{tabular}

Significant high incidence: ${ }^{*} \mathrm{p}<0.01,{ }^{* *} \mathrm{p}=0.01$ by chi square test

CCSs are generally defined as children who have survived more than 5 years after the cancer treatment. However, we included 2-year survivors in this study, because late effects such as growth retardation and hypogonadism should be managed as early as possible. This is a retrospective study, which may have a bias by selection at the time of reference. Although simple comparison is not appropriate in terms of the rate of complications, it is noteworthy to recognize the high incidence of endocrine complications, especially in CCSs associated with brain tumor, in the present study.

Growth retardation is one of the most common endocrinological complications emerged during cancer treatment and also after therapy in CCSs [8, 9, 23, 24]. It was also a frequent complication in the present study $(32 \%)$. The cause of the growth disturbance was multifactorial: inadequate nutrition, GHD, hypothyroidism, insufficient pubertal height gains due to hypogonadism, and corticosteroid therapy for chronic GVHD. Injury to the epiphyseal plate of the spine was also suspected, especially in patients treated with spinal irradiation and/or TBI. GH therapy has been reported to be beneficial for height gain. The height SD scores were improved by GH therapy in our study, as reported previously [25]. On the other hand, there are also concerns about the risk of recurrence of tumors and second neoplasm associated with GH therapy [26, 27]. We observed the relapse of germinoma during GH therapy in one patient, not included in the present study because of less than two years of follow-up. From a study of 361 GH-treated CCSs, and 12,963 non-GH-treated CCSs, Sklar et al. concluded that GH therapy does not appear to increase the risk of disease recurrence or death in $\mathrm{CCSs}$, although this therapy may increase the number of second tumors [28]. It is well known that higher circulating levels of IGF-1 are associated with an increased risk of malignancies especially colon cancer $[29,30]$. Thus, a regular monitoring of serum IGF-1 level is important for GH-treated CCSs.

Abnormal gonadal function is also a common problem, but it could not be evaluated before puberty. In male patients, it is sometimes difficult to recognize gonadal abnormalities from their appearance, because testosterone secretion is relatively reserved. In such cases, a high level of FSH $(>20 \mathrm{mIU} / \mathrm{ml})$ and decreased testis volume $(<10 \mathrm{ml})$ would be a key factor to make a diagnosis [16]. Pubertal girls showed ovarian damage with an extremely high level of FSH/LH, and they required hormonal supplementation. Only two adult patients, one male and one female, among 38 adult patients had healthy childbirth at present. We have not routinely performed sperm cryopreservation nor sperm evaluation. Fujita et al. reported that transplantation of spermatogonial stem cells isolated from leukemic mice restored fertility without seeding leukemia [31]. Although a few hopeful childbirths have been reported [32], preservation of fertility in women is a prolonged and important problem in CCSs [33]. Longer follow-up is needed in consideration with fertility.

As another long-term complication in CCSs, the risk of low BMD has been reported [34]. The leading method of assessing BMD is dual energy DXA, and age-, race-, and sex-specific reference curves can be 
used to help identify children with bone deficits and to monitor changes in the bone response to chronic diseases or therapies [35]. The standard of BMD in normal Japanese children may remain to be improved in view of small number of subjects; however, according to the report of Japanese childhood BMD [19], a decrease in BMD was observed in $42 \%$ cases in our study. The prevalence of osteopenia and osteoporosis in 98 CCSs was $31 \%$ and $11 \%$, respectively. In addition, vertebral fracture was observed in one patient with GVHD. Peak bone mass, achieved at the end of sexual development, is also reduced in our study. The reported etiology of bone loss in CCSs is multifactorial [36], and includes negative effects of the malignant disease itself, chemotherapeutic agents such as glucocorticoids and methotrexate, irradiation, GVHD, poor nutrition, prolonged physical inactivity, hypogonadism and growth hormone deficiency. Adequate bone mineral acquisition is especially important in children because it determines peak bone mass. Although our study has the limitation of small number of subjects, patients with a high risk of low BMD, such as GHD, gonadal dysfunction (especially female) and chronic GVHD, should be closely followed for a long time. Further studies are needed to evaluate the long-term effect on BMD in CCSs and to prevent pathological fractures later in life.

Multimodal therapy, consisting of chemotherapy, surgery and radiotherapy with or without HSCT, has recently been developed. Susceptibility to chemotherapy and radiotherapy differs depending on the age of patients and the dosage. Complicated side effects are serious problems, and it is not easy to predict each prognosis. The appearance of clinical or laboratory abnormalities take years. Especially gonadal dysfunction and growth disorders are more evident in adolescence. Annual testing before the initiation of puberty is thus strongly recommended, even though many of these patients are unaware of signs and symptoms of organ failure during childhood. Measurement of height and growth velocity and evaluation of pubertal stage should be performed at 3 to 6 months interval during growth period. Evaluation should be modified on a case-by-case basis even when both the diagnosis and therapy protocol are the same.

The present study did not include patients with less than 2 years of follow-up, patients not referred to endocrinologists or patients who had been lost to followup. The timing of patient reference from oncologist might have given a bias to selection of study population. Recently HSCT has been applied to an extended spectrum of diseases, including immunologic, genetic and metabolic nonhematological diseases, which should be evaluated in future study. Our study also has limitations because it is retrospective analysis of a cohort of a rather small number of patients in a single hospital; however, the therapy protocol, the condition for which oncologists refer to endocrinologists and clinical assessment are rather consistent among patients.

In summary, our study suggests that CCSs tend to develop late endocrine dysfunctions after cancer therapy. Lifetime medical surveillance and continuous follow-up are necessary for CCSs because treatmentrelated complications may occur during childhood and many years after the therapy as well. Endocrinologists should participate in the follow-up of these children in collaboration with pediatric oncologists.

\section{References}

1. Foundation for promotion of cancer research (2007) Cancer statistics in Japan - 2007. HYPERLINK "http:// ganjoho.ncc.go.jp/public/statistics/backnumber/2007 jp.html"

2. American Cancer Society (2007) Cancer Facts \& Figures 2007. HYPERLINK "http://www.cancer.org/docroot/ STT/content/STT_1x_Cancer_Facts_Figures_2007.asp"

3. National Institute for Health and $\bar{C}$ linical Excellence (NICE), Service Guidelines "Improving Outcomes in Children and Young People with Cancer" (2005) HYPERLINK "http://www.nice.org.uk"

4. Dickerman JD (2007) The late effects of childhood cancer therapy. Pediatrics 119: 554-568.

5. Oeffinger KC, Mertens AC, Sklar CA, Kawashima T, Hudson MM, Meadows AT, Friedman DL, Marina N, Hobbie W, Kadan-Lottick NS, Schwartz CL, Leisenring W, Robison LL; Childhood Cancer Survivor Study (2006) Chronic Health Conditions in Adult Survivors of Childhood Cancer. N Engl J Med 355: 1572-1582.

6. Geenen MM, Cardous-Ubbink MC, Kremer LC, van den Bos C, van der Pal HJ, Heinen RC, Jaspers MW, Koning CC, Oldenburger F, Langeveld NE, Hart AA, Bakker PJ, Caron HN, van Leeuwen FE (2007) Medical assessment of adverse health outcomes in long-term 
survivors of childhood cancer. JAMA 297: 2705-2715.

7. Bassal M, Mertens AC, Taylor L, Neglia JP, Greffe BS, Hammond S, Ronckers CM, Friedman DL, Stovall M, Yasui YY, Robison LL, Meadows AT, KadanLottick NS (2006) Risk of selected subsequent carcinomas in survivors of childhood cancer: a report from the Childhood Cancer Survivor Study. J Clin Oncol 24: 476-483.

8. Meacham L. (2003) Endocrine late effects of childhood cancer therapy. Curr Probl Pediatr Adolesc Health Care 33: 217-242.

9. Rutter MM, Rose SR (2007) Long-term endocrine sequelae of childhood cancer. Curr Opin Pediatr 19: 480-487.

10. Children's Oncology Group (2006) Long-Term Follow-Up Guidelines for Survivors of Childhood, Adolescent, and Young Adult Cancers. Version 2.0March 2006. HYPERLINK "http://www.survivorshipguidelines.org/"

11. SIGN Long term follow up of survivors of childhood cancer (2005) A national clinical guideline January 2004; Updated March 2005. HYPERLINK "http:// www.sign.ac.uk/pdf/sign76.pdf"

12. Practice Statement "Therapy Based Long Term Follow Up" (2nd Edition, 2005). HYPERLINK "http:// www.ukccsg.org.uk/public/followup/PracticeStatement/ index.html" UK Children's Cancer and Leukaemia Group (CCLG).

13. Masera G, Chesler M, Jankovic M, Eden T, Nesbit ME, Van Dongen-Melman J, Epelman C, Ben Arush MW, Schuler D, Mulhern R (1996) SIOP Working Committee on Psychosocial issues in pediatric oncology: guidelines for care of long-term survivors. Med Pediatr Oncol 27: 1-2.

14. Aslett H, Levitt G, Richardson A, Gibson F (2007) A review of long-term follow-up for survivors of childhood cancer. Eur J Cancer 43: 1781-1790.

15. Shinagawa $T$, Tomita $Y$, Ishiguro $H$, Matsumoto $M$, Shimizu T, Yasuda Y, Hattori K, Kubota C, Yabe H, Yabe M, Kato S, Shinohara O (2001) Final height and growth hormone secretion after bone marrow transplantation in children. Endocr J 48: 133-138.

16. Ishiguro H, Yasuda $Y$, Tomita $Y$, Shinagawa $T$, Shimizu $\mathrm{T}$, Morimoto T, Hattori $\mathrm{K}$, Matsumoto M, Inoue $\mathrm{H}$, Yabe H, Yabe M, Shinohara O, Kato S (2007) Gonadal shielding to irradiation is effective in protecting testicular growth and function in long-term survivors of bone marrow transplantation during childhood or adolescence. Bone Marrow Transplantation 39: 483-490.

17. Maeda N, Kato K, Matsuyama T, Kojima S, Ohyama K (2003) High-Dose Busulfan is a major risk factor for ovarian dysfunction in girls after stem cell transplantation. Clin Ped Endo 12: 13-18.

18. Osteoporosis Diagnostic Criteria Review Committee, Japanese Society for Bone and Mineral Research
(2001) Diagnostic criteria for primary osteoporosis: year 2000 revision. J Bone Miner Metab 19: 331-337.

19. Tanaka H (2005) Bone mineral density. Pediatrics of Japan 46 (suppl): 17-19 (In Japanese).

20. Schwartz CL, Hobbie WL, Constine LS, Ruccione KS, eds. (2005) Survivors of childhood and adolescent cancer: A multidisciplinary approach. Second edition. Germany: Springer-Verlag.

21. Haupt R, Spinetta JJ, Ban I, Barr RD, Beck JD, Byrne J, Calaminus G, Coenen E, Chesler M, D’Angio GJ, Eiser C, Feldges A, Gibson F, Lackner H, Masera G, Massimo L, Magyarosy E, Otten J, Reaman G, Valsecchi MG, Veerman AJ, Penn A, Thorvildsen A, van den Bos C, Jankovic M; International Berlin-FrankfurtMünster Study Group Early and Late Toxicity Educational Committee (I-BFM-SG ELTEC) (2007) Long term survivors of childhood cancer: cure and care. The Erice statement. Eur J Cancer 43: 1778-1780.

22. Sklar CA (2001) Endocrine complications of the successful treatment of neoplastic diseases in childhood. Growth Genetics and Hormones 17: 37-42.

23. Chow EJ, Friedman DL, Yasui Y, Whitton JA, Stovall M, Robison LL, Sklar CA (2007) Decreased adult height in survivors of childhood acute lymphoblastic leukemia: a report from the Childhood Cancer Survivor Study. J Pediatr 150: 370-375.

24. Sanders JE (2008) Growth and development after hematopoietic cell transplant in children. Bone Marrow Transplant 41: 223-227.

25. Gleeson HK, Stoeter R, Ogilvy-Stuart AL, Gattamaneni HR, Brennan BM, Shalet SM (2003) Improvements in final height over 25 years in growth hormone $(\mathrm{GH})$ deficient childhood survivors of brain tumors receiving GH replacement. J Clin Endocrinol Metab 88: 36823689.

26. Lawson Wilkins Pediatric Endocrine Society (LWPES) Writing Committee: Mark A. Sperling, Paul H. Saenger, Ray Hintz, Tom Wilson, and Susan R. Rose on behalf of the LWPES Executive Committee and the LWPES Drug and Therapeutics Committee (2002) Growth Hormone Treatment and Neoplasia-Coincidence or Consequence? J Clin Endocrinol Metab 87: 5351-5352.

27. Jenkins PJ, Mukherjee A, Shalet SM (2006) Does growth hormone cause cancer? Clin Endocrinol 64: 115-121.

28. Sklar CA, Mertens AC, Mitby P, Occhiogrosso G, Qin J, Heller G, Yasui Y, Robison LL (2002) Risk of disease recurrence and second neoplasms in survivors of childhood cancer treated with growth hormone: a report from the Childhood Cancer Survivor Study. $J$ Clin Endocrinol Metab 87: 3136-3141.

29. Cohen P, Clemmons DR, Rosenfeld RG (2000) Does the GH-IGF axis play a role in cancer pathogenesis? Growth Horm IGF Res 10: 297-305. 
30. Swerdlow AJ, Higgins CD, Adiard P, Preece MA (2002) Risk of cancer in patients treated with human pituitary growth hormone in the UK, 1959-85: a cohort study. Lancet 360: 273-277.

31. Fujita K, Ohta H, Tsujimura A, Takao T, Miyagawa Y, Takada S, Matsumiya K, Wakayama T, Okuyama A (2005) Transplantation of spermatogonial stem cells isolated from leukemic mice restores fertility without inducing leukemia. J Clin Invest 115: 1855-1861.

32. Donnez J, Dolmans MM, Demylle D, Jadoul P, Pirard C, Squifflet J, Martinez-Madrid B, Langendonckt AV (2004) Live birth after orthotopic transplantation of cryopreserved ovarian tissue. Lancet 364: 1405-1410.

33. Rogerio AL (2005) Potential options for preservation of fertility in women. $N$ Engl J Med 353: 64-73.

34. Sala A, Barr RD (2007) Osteopenia and cancer in children and adolescents: the fragility of success. Cancer 109: 1420-1431.

35. Kalkwarf HJ, Zemel BS, Gilsanz V, Lappe JM, Horlick M, Oberfield S, Mahboubi S, Fan B, Frederick MM, Winer K, Shepherd JA (2007) The Bone Mineral Density in Childhood Study: Bone Mineral Content and Density According to Age, Sex, and Race. J Clin Endocrinol Metab 92: 2087-2099.

36. Michaud LB, Goodin S. (2006) Cancer-treatmentinduced bone loss, part 1. Am J Health Syst Pharm 63: 419-430. 